\title{
Expression of Ganglioside GD2, as a Novel Cancer Stem Cell Marker in Breast Carcinomas
}

DOI: $10.21859 /$ mci-supp-31

\author{
Maryam Mansoori ${ }^{1}$, Alireza Mirzai ${ }^{2}$, Isa Abdi Rad ${ }^{3}$, Rahim \\ MahmoodLu ${ }^{4}$, Zahra Madjd ${ }^{1,2, *}$ \\ ${ }^{1}$ Department of Molecular Medicine, Faculty of Advanced Technologies in Medicine, \\ Iran University of Medical Sciences, Tehran, Iran \\ ${ }^{2}$ Oncopathology Research Center, Iran University of Medical Sciences (IUMS), \\ Hemmat Street (Highway), Next to Milad Tower, Tehran 14530-14496, Iran \\ ${ }^{3}$ Cellular and Molecular Research Center, Urmia University of Medical Sciences, \\ Urmia, Iran \\ ${ }^{4}$ Imam Khomeini Hospital, Urmia University of Medical Sciences, Urmia, Iran \\ * Corresponding author: Zahra Madjd, Department of Molecular Medicine, Faculty \\ of Advanced Technologies in Medicine, and Oncopathology Research Center, Iran \\ University of Medical Sciences, Tehran, Iran.E-mail:Zahra Madjd; zahra.madjd@ \\ yahoo.com
}

\section{Keywords:}

Breast Cancer

Cancer Stem Cell

GD2

Marker

\begin{abstract}
Introduction: Cancer stem cells (CSCs) are attractive for many researchers because of their diagnostic, prognostic and therapeutic implications. Here, we aim to investigate the local expression of Ganglioside GD2, as the recently proposed breast CSC (BCSC) marker, through the assessment of GD2 synthase mRNA, the key enzyme involved in GD2 synthesis.
\end{abstract}

Materials and Methods: Fresh tumor tissues along with normal adjacent tissues from 65 patients who underwent surgery for breast cancer were collected. Total RNAs of tumoral and normal tissue samples were extracted and analyzed using real-time PCR. The mRNA expression pattern of GD2 was compared in tumoral and control tissue samples using $\Delta \Delta$ Ct method. The relevance of GD2 synthase level with clinical characteristics of the patients was also evaluated.

Results: The expression level of GD2 synthase was significantly higher in breast cancer tissue samples compared to normal adjacent tissues (4.92-fold change, $\mathrm{P}<0.001)$. GD2 synthase expression was also significantly higher in advanced grades tumor ( 2 and 3 ) $(\mathrm{P}=0.001)$ and in patients at stages III and IV compared to stages I and II $(\mathrm{P}=0.001)$. Whereas, the correlation between GD2 synthase and other clinicopathologic data was not statistically significant, such as for age $(\mathrm{P}=1.000)$, type of carcinoma $(\mathrm{P}=0.934)$, lymphatic invasion $(\mathrm{P}=0.139)$ and tumor size $(\mathrm{P}=0.066)$.

Conclusions: The higher expression of GD2 synthase in tumoral tissue samples of breast cancer patients further potentiates it as a BCSC marker which could be used for clinical implications such as diagnosis and prognosis of breast cancer. 